\title{
EEG Based Biometric Authentication Using New Spectral Features
}

\author{
Isao Nakanishi, Sadanao Baba and Chisei Miyamoto \\ Tottori University, Japan \\ E-mail: nakanishi@ele.tottori-u.ac.jp Tel: +81-857-31-5132
}

\begin{abstract}
From the viewpoint of user management, continuous authentication is effective. However, general biometrics such as fingerprint, iris, vein, and so on are not suitable for the continuous verification since they require conscious presentation of biometric data. As unconscious biometrics, to use a brain wave (electroencephalogram: EEG) has been proposed but conventional approaches require heavy computational load for feature extraction and verification and it becomes a problem for practical applications. In this paper, we propose new features: the concavity and convexity of spectral distribution in the alpha band and propose to fuse them with a spectral variance in verification. The proposed methods are achieved with low computational load. In our experiments using 23 subjects, EER of $11 \%$ is obtained and it is comparable with the performance of conventional approaches.
\end{abstract}

\section{INTRODUCTION}

A number of biometrics are being proposed and used in various authentications. Each biometrics is evaluated in seven factors: universality, uniqueness, permanence, measurability, performance, acceptability, and circumvention [1]. Particularly, it is well known that fingerprint achieves higher performance in identification or verification. However, it has been reported that authentication systems based on the fingerprint were circumvented by fake fingers [2]. The reason is that the fingerprint is revealed on the body surface.

From a viewpoint of the tolerability to such circumvention, the biometrics kept in the body such as a vein is better. However, there was a report that even the authentication system using the vein accepted artifacts in enrollment and verification. This is due to lack of liveness detection which examines whether an object is a part of a living body. The liveness detection scheme is necessary for protecting the biometric authentication system from spoofing using artifacts.

Furthermore, conventional biometric authentication systems assume the applications based on one-time-only authentication such as access control, banking, passport control, and so on. On the other hand, from the viewpoint of user management, one-time-only authentication is low-security. After the authentication by a genuine user, even if he/she is switched to an imposer, the authentication system could not detect such spoofing. In order to cope with this problem, continuous authentication is needed and it requires unconscious biometrics in which the user is without regard for presenting his/her biometrics. The fingerprint and iris are not suitable for the continuous authentication since they ask users to keep presenting their biometrics during authentication. As unconscious biometrics, face, ear, and gate are famous but the face and ear can be imitated using artifacts and the gate limits applications.

It has been proposed to use a brain wave (electroencephalogram: EEG) as a biometrics [3]-[13]. The brain wave is generated by the activities of neurons in a brain cortex, that is, it is kept in the body; therefore, it is effective for anti-circumvention. Of course, the brain wave possesses the function of liveness detection since it is generated by only live human beings.

However, conventional approaches have not specified their applications. Almost they required many electrodes (sensors) and long measuring time. In addition, some of them adopted AR modeling for feature extraction and neural-networks (a learning algorithm) for verification. Their heavy computational load is not suitable for practical applications while they achieved the verification rate of over $90 \%$.

For reducing the computational load, we adopted shorttime measurement using a single sensor and proposed features based on the spectrum analyses of the brain wave, especially the alpha wave [14]. In this paper, we propose a new feature based on the convexity of spectral distribution. Verification is done based on a linear combination of these features.

\section{Measurement of Brain WaVe}

\section{A. Brain Wave}

Electrical changes macroscopically detected on the scalp using an electrode are defined as a brain wave, which is illustrated in a figure called Electroencephalogram (EEG) in which a horizontal axis is of time and a vertical one is of voltage. The brain wave is categorized in five bands: delta $(0.5-3 \mathrm{~Hz})$, theta $(4-7 \mathrm{~Hz})$, alpha $(8-13 \mathrm{~Hz})$, beta $(14-30 \mathrm{~Hz})$ and gamma $(>30 \mathrm{~Hz})$, respectively.

In conventional studies, the authentication using the alpha wave has been evaluated with a pioneer spirit [3]-[6]. The alpha wave is detectable when human beings are relaxed with closed eyes. In this paper we also use the alpha wave in authentication.

\section{B. Measurement System and Condition}

In conventional studies, the alpha wave was measured by using a medical-use electroencephalograph, which requires subjects to set a number of electrodes on the scalp [3]-[6], [9]. However, it is inconvenience for them when assuming practical applications. In addition, multichannel measurement increases 


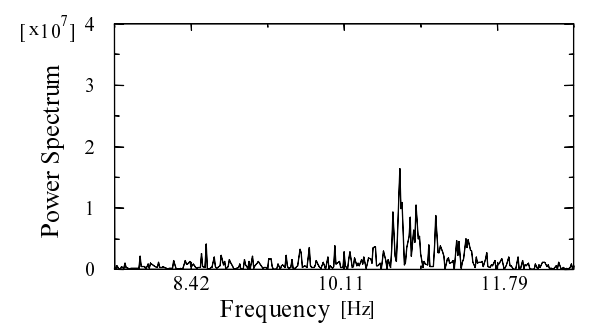

(a)

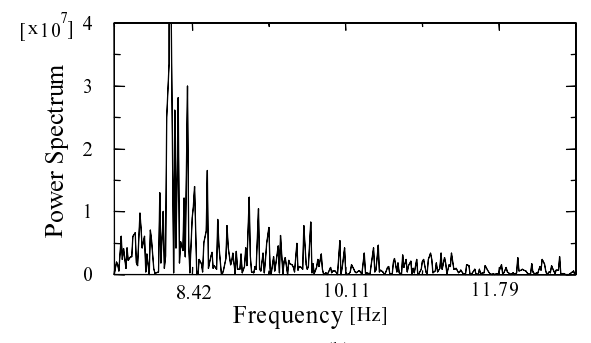

(b)

Fig. 1. Power spectra of two subjects in the alpha band.

the number of processing data, that is, it needs heavy computational load. Resultingly, the multichannel measurement is not suitable for practical applications while it could provide higher authentication accuracy.

In this paper, we use a consumer-use electroencephalograph which has only one electrode (single-channel). The specifications of the electroencephalograph are as follows: frequency range is $4-24 \mathrm{~Hz}$, sampling rate is $128 \mathrm{~Hz}$, voltage range is 5-80 $\mu V_{p p}$, and measurement accuracy is \pm 10 .

This instrument consists of a head-band sensor and a main unit. By using the head-band, a single electrode is set on the frontal region of a head which corresponds to Fp1 in the international 10/20 system. The main unit includes an A/D convertor and an amplifier and is connected with a Windowsbased PC by RS-232C. The measurement is controlled by a software tool, and measured data are saved and then analyzed in the computer.

Subjects are requested to sit on a chair at rest with eyeclosed in a dim and quiet room since it is a suitable condition for detecting the alpha wave.

\section{ANALYSIS OF BRAIN WAVE}

As an orthodox method, we adopt the spectrum analysis based on fast Fourier transform (FFT). Additionally, the spectrum analysis makes it easy to filter the spectrum in the alpha band.

Figure 1 shows power spectra of two subjects in the alpha band. The spectral distribution is unique, that is, the interclass variation of the power spectrum in the alpha band is large. Therefore, the spectral analysis is effective for feature extraction from the alpha wave.

Figure 2 shows intra-class variation in the alpha band of two subjects. Individual power spectra described as solid lines are the same as those in Fig. 1. Each dashed line is obtained by averaging 10 power spectra of each subject. From these

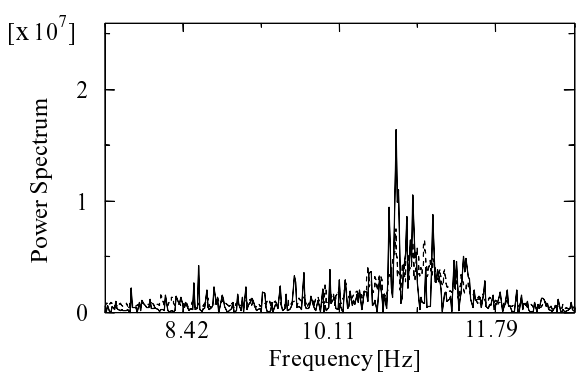

(a)

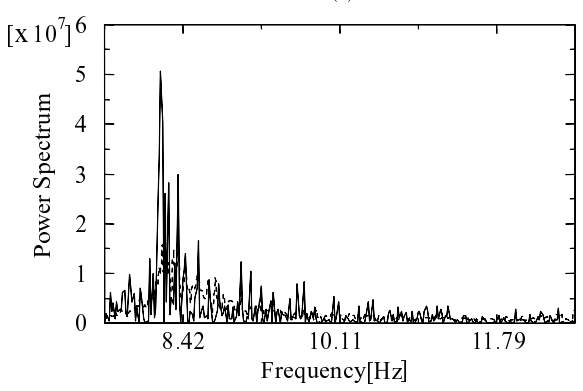

(b)

Fig. 2. Comparison of a power spectrum with a mean spectrum.

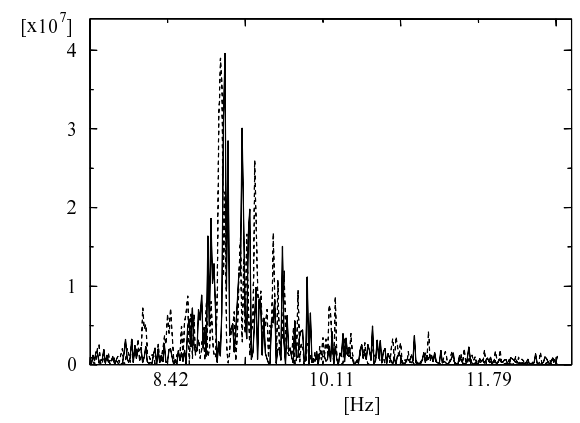

Fig. 3. Long-term variation of the power spectrum in the alpha band.

comparisons, it clear that the intra-class variation of the power spectrum in the alpha band is relatively small comparing with the inter-class variation shown in Fig. 1.

The comparison of the power spectrum (the solid line) with that after about one year (the dashed line) is shown in Fig. 3. This suggests that the long-term variation of the power spectrum in the alpha band is small.

As examined above, each individual characteristic of the power spectrum in the alpha band is different from those of others. On the other hand, the intra-class and long-term variations in individual spectra are relatively small. As a result, it is expected that the power spectrum of the brain wave is effective for person authentication.

\section{VERIFICATION USING BRAIN WAVE}

In this section, we examine how to extract individual features from a power spectrum of the brain wave and how to verify individuals using them.

The conventional studies utilized AR modeling for feature detection and/or learning algorithms such as neural-networks 


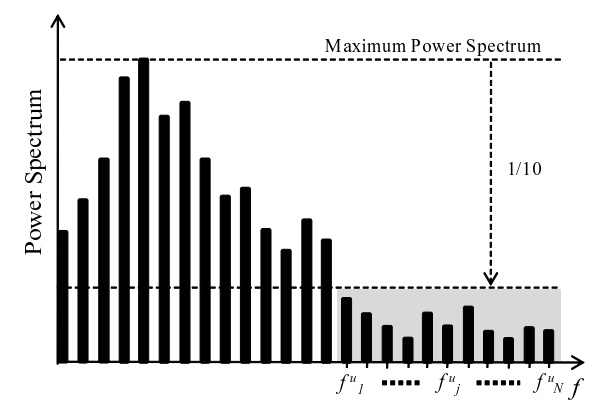

Fig. 4. Definition of the concavity of spectral distribution.

for verification/identification [3]-[6], [9], [13]. However, these methods need heavy computational load while they are effective for achieving higher authentication accuracy. From a viewpoint of practical applications, it is desired to reduce the computational load for real-time processing.

\section{A. Feature Extraction}

We have proposed spectral features based on the concavity of spectral distribution and to fuse it with a spectral variance [14]. In this paper, we propose a new spectral feature based on the convexity of spectral distribution and combine it with conventional features in verification.

The definition of the concavity of spectral distribution is shown in Fig. 4. First, the maximum of the power spectrum is detected and then its tenth part is calculated and adopted as a criterion. Then, frequencies of which power spectral values are under the criterion are squared and then summed as

$$
F_{u}=\sum_{j=1}^{N}\left(f_{j}^{u}\right)^{2}
$$

where $f_{j}^{u}(j=1,2, \cdots, N)$ is frequencies under the criterion.

$F_{u}$ is regarded as a feature representing the concavity of spectral distribution.

As a feature which represents how the broadening of spectral distribution is, we adopt a spectral variance assuming the spectrum in the alpha band is normally-distributed as shown in Figs. 1-3.

$$
\sigma^{2}=\frac{1}{L} \sum_{k=1}^{L}\left(s_{k}-\bar{s}\right)^{2}
$$

where $s_{k}(k=1,2, \cdots, L)$ are power spectral values in the alpha band and $\bar{s}$ is their mean value.

In addition to the above features, the convexity of spectral distribution is another important feature for distinguishing individuals as shown in Fig. 1. The convexity of spectral distribution is defined in the following manner.

Firstly, the power spectral values in the alpha band are ranked and then the values and the frequencies of the top three are respectively averaged. This process is illustrated as (a) in Fig. 5. Let the frequencies and the power spectral values of the top three be $f_{1}, f_{2}, f_{3}$ and $s_{1}, s_{2}, s_{3}$ respectively, their

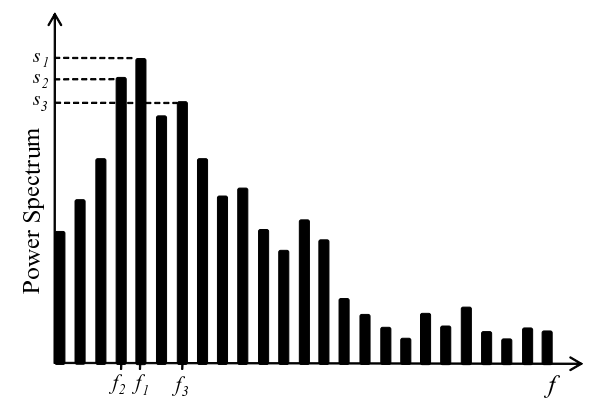

(a)

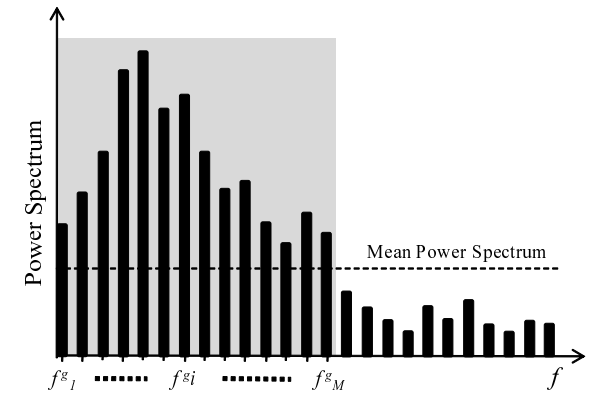

(b)

Fig. 5. Definitions of the convexity of spectral distribution.

mean values are given by

$$
F_{m}=\left(f_{1}+f_{2}+f_{3}\right) / 3 \quad S_{m}=\left(s_{1}+s_{2}+s_{3}\right) / 3
$$

Next, assuming spectral values which are greater than the mean power spectrum are $f_{i}^{g}(i=1,2, \cdots, M)$ as shown in Fig. 5(b), their summation is given by

$$
F_{g}=\sum_{i=1}^{M} f_{i}^{g}
$$

In this paper, $F_{m}, S_{m}$ and $F_{g}$ are regarded as features which represent the convexity in spectral distribution.

\section{B. Verification}

The similarities in the concavity of spectral distribution and the spectral variance are examined in

$$
\begin{aligned}
\text { Conc } & =\left|F_{u}-F_{u}^{t}\right| \\
\text { Var } & =\left|\sigma^{2}-\sigma^{2 t}\right|
\end{aligned}
$$

where $F_{u}^{t}$ and $\sigma^{2 t}$ are the templates of $F_{u}$ and $\sigma^{2}$, respectively [14].

The similarity in the convexity of spectral distribution is examined as

$$
\begin{aligned}
\text { Conv }=\lambda_{1} & \left\{\lambda_{2}\left|F_{m}-F_{m}^{t}\right|+\left(1-\lambda_{2}\right) \kappa\left|S_{m}-S_{m}^{t}\right|\right\} \\
& +\left(1-\lambda_{1}\right)\left|F_{g}-F_{g}^{t}\right|
\end{aligned}
$$

where $F_{m}^{t}, S_{m}^{t}$ and $F_{g}^{t}$ are the templates of $F_{m}, S_{m}$ and $F_{g}$, respectively. $\kappa$ is a constant for adjusting a spectral dimension to a frequency one. $\lambda_{1}, \lambda_{2}$ are weights for combining three features. 


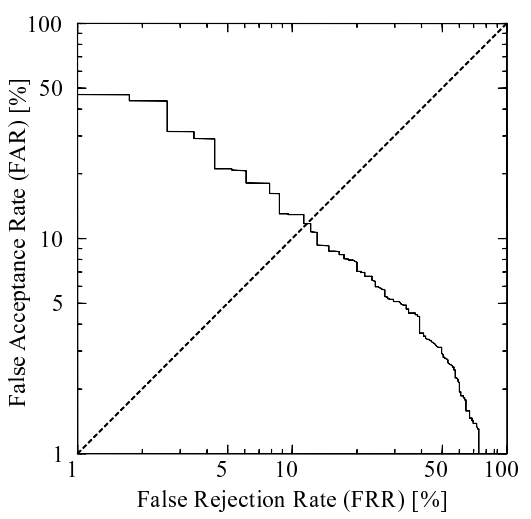

Fig. 6. A ROC curve.

Total similarity is defined by combining the above similarities.

$$
\mathrm{TS}=\delta \text { Conv }+(1-\delta)\{\gamma \text { Conc }+(1-\gamma) \operatorname{Var}\}
$$

where $\delta$ and $\gamma$ are the weights for determining the contribution ratios of three features.

When the TS is smaller than a threshold, the verification data are decided to be identical to the template and then the subject is verified to be genuine.

\section{EXPERIMENTS}

In order to examine the proposed features and verification algorithm, we carried out experiments in authentication.

The number of subjects was 23. All were healthy male around twenty and seated at rest with closed eyes in a silent room. An EEG signal (brain wave) was recorded using the single-channel electroencephalograph described in Sect. II-B during continuous three minutes and the data for the last one minute was used, which corresponded to 7680 sampled data and these were used for FFT analysis.

10 EEG signals were recorded from each subject on the same day. Among the 10 EEG data, five data were used for generating a template and the rest five data were used for verification. The template was obtained by averaging the five power spectral values at the same frequency bin.

Constants: $\lambda_{1}, \lambda_{2}, \delta$ and $\gamma$ for the verification were set to $0.3,0.2,0.3$ and 0.5 , respectively. These were determined by trial and error.

Figure 6 shows a ROC curve which plots False Rejection Rate (FRR) versus False Acceptance Rate (FAR). From this curve, Equal Error Rate (EER) is about $11 \%$ and it is comparable with those of conventional studies.

In the conventional studies, the comparable performance was achieved based on using many sensors, long measuring time, the modeling for feature extraction and/or the learning algorithm for verification. On the other hand, the proposed feature extraction and verification methods are simple and based on the usage of a single sensor. It is obvious that the computational load of the proposed methods is less than those of the conventional studies.

\section{CONCLUSIONS}

In the authentication using the brain wave, we proposed new features: the concavity and convexity of spectral distribution in the alpha band and proposed to fuse them with a spectral variance in verification. The proposed methods use only a single sensor; therefore, they are achieved with low computational load. In our experiment using 23 subjects, EER of $11 \%$ was obtained and it was comparable with the performance in conventional studies which required heavy computational load.

Assuming practical applications, to examine verification performance in eye-opening condition with doing some mental task is a future problem.

\section{ACKNOWLEDGEMENT}

A part of this work was supported by the Support Center for Advanced Telecommunications Technology Research, Foundation (SCAT) in Japan.

\section{REFERENCES}

[1] A. Jain, R. Bolle and S. Pankanti, BIOMETRICS Personal Identification in Networked Society, Kluwer Academic Publishers, Massachusetts, 1999.

[2] T. Matsumoto, H. Matsumoto, K. Yamada and S. Hoshino, "Impact of Artificial "Gummy" Fingers on Fingerprint Systems," Proc. of SPIE, vol. 4677, pp. 275-289, Jan. 2002.

[3] M. Poulos, M. Rangoussi, V. Chrissikopoulos, A. Evangelou, "Person Identification Based on Parametric Processing of the EEG," Proc. of the 6th IEEE Int. Conf. on Electronics, Circuits and Systems, vol.1, pp.283-286, 1999.

[4] M. Poulos, M. Rangoussi, and N. Alexandris, "Neural Networks Based Person Identification Using EEG Features," Proc. of ICASSPf99, pp. 1117-1120, 1999.

[5] M. Poulos, M. Rangoussi, V. Chissikopoulus, and A. Evangelou, "Parametric Person Identification from the EEG Using Computational Geometry," Proc. of the 6th IEEE International Conference on Electronics, Circuits and Systems, pp. 1005-1008, 1999.

[6] R. B. Paranjape, J. Mahovsky, L. Benedicent, and Z. Koles, "The Electroencephalogram as a Biometric," Proc. of 2001 Canadian Conference on Electrical and Computer Engineering, vol. 2, pp. 1363-1366, 2001.

[7] K. V. R. Ravi and R. Palaniappan, "Recognition Individuals Using Their Brain Patterns," Proc. of the 3rd International Conference on Information Technology and Applications, 2005.

[8] R. Palaniappan, "Identifying Individuality Using Mental Task Based Brain Computer Interface," Proc. of the 3rd International Conference on Intelligent Sensing and Information Processing, pp. 239-242, 2005.

[9] G. Mohammadi, P. Shoushtari, B. M. Ardekani, M. B. Shamsollahi, "Person Identification by Using AR Model for EEG Signals," Proc. of World Academy of Science, Engineering and Technology, vol. 11, no. 2, pp. 281-285, Feb. 2006

[10] S. Marcel and J. R. Millan, "Pearson Authentication Using Brainwaves (EEG) and Maximum A Posteriori Model Adaption," IEEE Trans. on Pattern Analysis and Machine Intelligence, vol. 29, no. 4, pp. 743-748, Apr. 2007.

[11] R. Palaniappan, D. P. Mandic, "Biometrics from Brain Electrical Activity: A Machine Learning Approach," IEEE Trans. on Pattern Analysis and Machine Intelligence, vol. 29, no. 4, pp. 738-742, Apr. 2007.

[12] G. K. Singhal and P. RamKumar, "Person Identification Using Evoked Potentials and Peak Matching," Proc. of 2007 Biometric Symposium, 2007.

[13] A. Riera, A. Soria-Frish, M.Caparrini,C.Grau, and G.Ruffini, "Unobtrusive Biometrics Based on Electroencephalogram Analysis," EURASHIP Journal on Advances in Signal Processing, vol. 2008, pp.1-8, 2008.

[14] C. Miyamoto, S. Baba, and I. Nakanishi, "Biometric Person Authentication Using New Spectral Features of Electroencephalogram (EEG)," Proc. of 2008 International Symposium on Intelligent Signal Processing and Communication Systems (ISPACS2008), pp. 312-315, Nov. 2008. 